\title{
Caracterização do perfil clínico e nutricional de pacientes renais em tratamento hemodialítico em Belém, Pará
}

\author{
Characterization of the clinical and nutritional profile of renal patients hemodialytic treatment in \\ Belém, Pará \\ Caracterización del perfil clínico y nutricional de pacientes renales en tratamiento hemodialitico en \\ Belém, Pará
}

\author{
Alicía Gleides Fontes Gonçalves \\ ORCID: https://orcid.org/0000-0002-3148-0057 \\ Universidade Federal do Pará, Brasil \\ E-mail: aliciafontes.nutri@gmail.com \\ Emily de Cássia Cruz dos Santos \\ ORCID: https://orcid.org/0000-0001-8289-1561 \\ Universidade Federal do Pará, Brasil \\ E-mail: millycassie7@gmail.com \\ Aldair da Silva Guterres \\ ORCID: https://orcid.org/0000-0002-8388-0116 \\ Universidade Federal do Pará, Brasil \\ E-mail: guterres39@hotmail.com \\ Rosileide de Souza Torres \\ ORCID: https://orcid.org/0000-0002-8168-3434 \\ Universidade Federal do Pará, Brasil \\ E-mail: rosileide2@gmail.com \\ Camila Yohanna Lira Sousa \\ ORCID: https://orcid.org/0000-0003-0193-1600 \\ Universidade Estadual do Maranhão, Brasil \\ E-mail: nutricamilalira@gmail.com \\ Raissa Castro Ribeiro \\ ORCID: https://orcid.org/0000-0002-2343-2952 \\ Centro Universitário do Pará, Brasil \\ E-mail: raissa_cas@hotmail.com \\ Lisandra Rodrigues de Medeiros \\ ORCID: https://orcid.org/0000-0002-1376-0119 \\ Universidade do Estado do Pará, Brasil \\ E-mail: lisandramedeiros.1@gmail.com \\ Samara da Silva Queiroz \\ ORCID: https://orcid.org/0000-0002-1842-6259 \\ Universidade Federal do Pará, Brasil \\ E-mail: samarasila_07@hotmail.com \\ Daniela Lopes Gomes \\ ORCID: https://orcid.org/0000-0002-0092-3396 \\ Universidade Federal do Pará, Brasil \\ E-mail: danielagomes@ufpa.br \\ Manuela Maria de Lima Carvalhal \\ ORCID: https://orcid.org/0000-0003-1397-0471 \\ Universidade Federal do Pará, Brasil \\ E-mail: manuela.carvalhall@gmail.com
}

\begin{abstract}
Resumo
Introdução: A doença renal crônica associa-se à alterações clínicas e nutricionais, decorrentes da própria patologia, devido as alterações homeostáticas e metabólicas que a disfunção renal causa, e do tratamento. Objetivo: Caracterizar o perfil clínico e nutricional de pacientes renais crônicos em tratamento hemodialítico. Metodologia: Estudo descritivo, transversal, quantitativo, analítico e documental, realizado com 50 pacientes. Foram coletados dados socioeconômicos, demográficos, clínicos e bioquímicos, sendo este último realizado após hemodiálise. Posteriormente foi realizada avaliação antropométrica e de consumo alimentar (recordatório 24 horas). O projeto foi aprovado pelo comitê de ética em pesquisa (parecer $n^{\circ}$ 2.970.945). Resultados: Observou-se equivalência de gêneros, com média de idade de $57 \pm 18,8$ anos. As comorbidades mais frequentes foram hipertensão arterial sistêmica (82\%) e diabetes mellitus tipo 2 (48\%). Na avaliação bioquímica os valores de creatinina, hemoglobina, cálcio sérico e hematócrito encontravam-se reduzidos,
\end{abstract}


ureia, potássio sérico, ferritina e glicemia estiveram elevados. verificou-se prevalência de eutrofia na análise de índice de massa corporal (IMC) $(56 \%)$ e baixa musculatura na análise de circunferência do braço (CB). O ganho de peso interdialítico (GPID) apresentou média de 2,5\% $( \pm 0,8)$ e esteve negativamente relacionado com a albumina, e apresentou correlação positiva com os níveis séricos de potássio, fósforo e cálcio e potássio dietético. O IMC apresentou correlação positiva com os níveis séricos de hematócrito e glicemia. Conclusão: Houve alteração no estado nutricional, bioquímico e dietético dessa população, observa-se que a associação entre esses indicadores possibilita um resultado mais completo sobre o estado nutricional de pacientes renais crônicos, possibilitado a correta intervenção nutricional.

Palavras-chave: Doença renal; Hemodiálise; Antropometria; Consumo alimentar.

\begin{abstract}
Introduction: Chronic kidney disease is associated with clinical and nutritional changes, resulting from the pathology itself, due to the homeostatic and metabolic changes that kidney dysfunction causes, and the treatment. Objective: To characterize the clinical and nutritional profile of patients with chronic kidney disease undergoing hemodialysis. Methodology: Descriptive, cross-sectional, quantitative, analytical and documentary study, carried out with 50 patients. Socioeconomic, demographic, clinical and biochemical data were collected, the latter being performed after hemodialysis. Subsequently, anthropometric and food consumption assessments were performed (24-hour recall). The project was approved by the research ethics committee (opinion No. 2,970,945). Results: Gender equivalence was observed, with a mean age of $57 \pm 18.8$ years. The most frequent comorbidities were systemic arterial hypertension $(82 \%)$ and type 2 diabetes mellitus $(48 \%)$. In the biochemical evaluation, the values of creatinine, hemoglobin, serum calcium and hematocrit were reduced, urea, serum potassium, ferritin and blood glucose were high. there was a prevalence of eutrophy in the analysis of body mass index (BMI) (56\%) and low musculature in the analysis of arm circumference (AC). Interdialytic weight gain (IDWG) averaged $2.5 \%( \pm 0.8)$ and was negatively related to albumin, and positively correlated with serum levels of potassium, phosphorus, calcium and dietary potassium. BMI showed a positive correlation with serum hematocrit and blood glucose levels. Conclusion: There was a change in the nutritional, biochemical and dietary status of this population, it is observed that the association between these indicators allows a more complete result on the nutritional status of chronic kidney patients, enabling the correct nutritional intervention.

Keywords: Chronic kidney; Hemodialysis; Anthropometry; Food consumption.
\end{abstract}

\title{
Resumen
}

Introducción: La enfermedad renal crónica se asocia a cambios clínicos y nutricionales, derivados de la propia patología, debido a los cambios homeostáticos y metabólicos que provoca la disfunción renal, y al tratamiento. Objetivo: Caracterizar el perfil clínico y nutricional de pacientes con enfermedad renal crónica en hemodiálisis. Metodología: Estudio descriptivo, transversal, cuantitativo, analítico y documental, realizado con 50 pacientes. Se recogieron datos socioeconómicos, demográficos, clínicos y bioquímicos, siendo este último realizado después de la hemodiálisis. Posteriormente, se realizaron valoraciones antropométricas y de consumo de alimentos (recordatorio de 24 horas). El proyecto fue aprobado por el comité de ética en investigación (dictamen $n^{\circ}$ 2.970.945). Resultados: Se observó

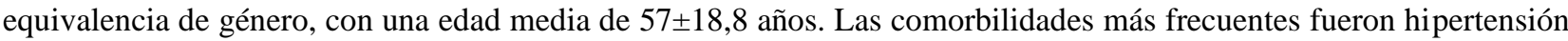
arterial sistémica (82\%) y diabetes mellitus tipo 2 (48\%). En la evaluación bioquímica se encontraron reducidos los valores de creatinina, hemoglobina, calcio sérico y hematocrito, urea, potasio sérico, ferritina y glicemia elevados. hubo prevalencia de eutrofia en el análisis del índice de masa corporal (IMC) (56\%) y baja musculatura en el análisis del perímetro braquial (CA). El aumento de peso interdialítico (IDWG) promedió $2,5 \%( \pm 0,8)$ y se relacionó negativamente con la albúmina y positivamente con el potasio sérico, el fósforo, el calcio y el potasio de la dieta. El IMC mostró una correlación positiva con el hematocrito sérico y la glucosa en sangre. Conclusión: Hubo un cambio en el estado nutricional, bioquímico y dietético de esta población, se observa que la asociación entre estos indicadores permite un resultado más completo sobre el estado nutricional de los pacientes renales crónicos, posibilitando la correcta intervención nutricional.

Palabras clave: Enfermedad renal; Hemodiálisis; Antropometría; Consumo de comida.

\section{Introdução}

O Brasil vem passando por uma transição nutricional, marcada pelo alto consumo de alimentos processados e ultraprocessados, em detrimento da baixa aquisição e ingestão de alimentos in natura, associado ainda a hábitos de vida não saudáveis, aumentando a ocorrência de doenças e agravos não transmissíveis (DANT's) (Sesso et al., 2017).

Dentre as principais DANT's, destaca-se a doença renal crônica (DRC), que manifesta importante ascendência nas últimas décadas, apresentando-se como problema de saúde pública no país, estando associada à elevada taxa de morbimortalidade (Sesso et al., 2017). O desenvolvimento da DRC é influenciado por diversos fatores de riscos, tais como: tabagismo, etilismo, excesso de peso, hipercolesterolemia, sedentarismo e hábitos dietéticos inadequados (Alvarenga et al., 2017). Além disto, a presença de 
outras DANT's tais como, hipertensão arterial sistêmica (HAS) (35\% das causas), diabetes mellitus tipo 2 (DM2) (28,5\%) e glomerulonefrites (11,5\%), são importantes potencializadores do seu desenvolvimento (Sesso et al., 2017).

É uma doença caracterizada pela perda progressiva e irreversível da função renal, sendo importante influenciadora da qualidade de vida (QV), impossibilitando o organismo do indivíduo de realizar as funções imprescindíveis para homeostase, sendo o transplante renal o tratamento mais recomendado e na sua ausência torna-se indispensável a utilização de terapias substitutivas (Butyn et al., 2021).

A hemodiálise é a terapia substitutiva mais eficaz, entretanto, apesar de necessária, apresenta consequências nutricionais, sendo comum os pacientes apresentarem prevalência, mesmo que contraditória, de desnutrição ou sobrepeso/obesidade. Além disso, pode ser observada uma relação da taxa de mortalidade com marcadores de desnutrição, especialmente os níveis séricos de fósforo, albumina, cálcio, dentre outros (Oliveira et al., 2021).

Assim a nutrição é uma das áreas multiprofissionais mais importantes para os cuidados dos pacientes com DRC, estando interligada a expectativa de vida dos pacientes em tratamento hemodialítico, para auxiliar na terapia específica e na determinação das necessidades do paciente (Oliveira et al., 2021; Butyn et al., 2021).

Devido a crescente ocorrência da DRC faz-se imprescindível a realização de estudos de monitoramento dos marcadores clínicos, nutricionais e alimentares desses, a exposição e discussão dessas informações poderá agregara a composição de dados nacionais oferecendo uma visão mais panorâmica das características clinicas, nutricionais e alimentares desses pacientes fornecendo subsidio para estudos futuros sobre as características e divergências desse grupo de pacientes (Butyn et al., 2021). Desta forma, o presente trabalho tem como objetivo caracterizar o perfil clínico e nutricional de pacientes renais crônicos em tratamento hemodialítico.

\section{Metodologia}

Trata-se de um estudo descritivo, transversal, quantitativo, analítico e documental realizado com 50 pacientes portadores de doença renal crônica que estavam realizando tratamento ambulatorial no Serviço de Terapia Renal Substitutiva (STRS) de um Hospital de referência na cidade de Belém no estado do Pará, no período de janeiro a novembro de 2019. Foram incluídos indivíduos que apresentaram aptidão mental para responder os formulários, aptidão física para realização da avaliação antropométrica e que aceitaram participar do estudo, ao assinarem o Termo de Consentimento Livre e Esclarecido (TCLE). Foram excluídos, pacientes transplantados, edemaciados, amputados, cadeirantes, e com intolerância ou alergia alimentar, aqueles que apresentaram indisponibilidade de informações necessárias para o estudo nos prontuários. As coletas iniciaram-se somente após aprovação do comitê de ética em pesquisa da FHCGV sob o parecer nº 2.970.945.

Foi realizada avaliação antropométrica considerando análise do índice de massa corporal (IMC), circunferência do braço (CB) e ganho de peso interdialítico (GPID). O IMC foi obtido pela relação entre o peso (seco) (Kg) e altura ao quadrado (m²). O peso foi aferido em balança plataforma marca Micheletti®, modelo (MIC- Cadeirante) com capacidade de até $600 \mathrm{~kg}$ e precisão de 100 g. A estatura foi verificada por estadiômetro portátil Personal Caprice da Sanny® tendo a haste de medição dobrável e seu tripé de apoio retrátil com extensão de 2,5m, escala de $0,1 \mathrm{~cm}$. respectivamente. A CB foi aferida com uma fita métrica flexível, no ponto médio entre o acrômio e o olecrano e classificado de acordo com os valores de percentil de referência. Para o cálculo do GPID, utilizou-se a fórmula: GPID = Peso pré-Hemodialise (HD) (atual) - peso pós-HD (anterior). O GPID relativo (GPIDr) foi obtido pela razão entre o GPID $(\mathrm{Kg})$ e o respectivo peso seco, utilizando a fórmula: GPIDr $=G P I D(k g) \div p e s o$ seco $x$ 100. Considerou-se normal um GPIDr entre 3 a $5 \%$ em relação ao peso seco.

Os dados socioeconômicos demográficos, clínicos e parâmetros bioquímicos foram coletados mediante consulta do prontuário do paciente. Para caracterização clínica, verificou-se a presença de comorbidades tais como, DM2, HAS, cardiopatias, câncer e outras, categorizadas em presente ou ausente. 
Em relação à avaliação bioquímica, foram considerados os exames realizados pelo laboratório terceirizado do hospital, observou-se dosagens séricas de albumina, ferritina, creatinina, hematócrito, hemoglobina, ferro, potássio, fósforo, cálcio, glicemia, proteínas totais, ureia pré e ureia pós hemodiálise.

Para avaliação quantitativa do consumo alimentar, foi aplicado o Recordatório Alimentar de 24 horas (R24h), com coleta de três dias alternados de uma mesma semana. As quantidades alimentares obtidas foram inseridas no Software de Nutrição Dietbox $^{\circledR}$. Foram avaliadas as quantidades de calorias (Kcal) e ingestões absolutas dos macronutrientes (g) (carboidratos, proteínas e lipídios) e micronutrientes (mg) (cálcio, potássio, fósforo e sódio).

Foi realizada a estatística descritiva dos dados, expressa por meio de medidas de tendência central e dispersão. O teste de normalidade kolmogorov-Smirnov foi utilizado para verificar a distribuição das variáveis. Para a análise de correlação bivariada foi aplicado o teste de correlação de Pearson e de Spearman conforme a distribuição simétrica e assimétrica dos dados. Foi considerado nível de significância estatística de 5\%. Utilizou-se o software Statistical Package for Social Science, versão 21.0 (SPSS Inc, Chicago, IL, USA) para as análises.

\section{Resultados}

Foram avaliados 50 pacientes, dos quais observou-se que 50\% ( $\mathrm{n}=25)$ era do sexo masculino e $50 \%(\mathrm{n}=25)$ do sexo feminino. A média de idade obtida foi de 57 anos $( \pm 18,8)$. A maioria dos indivíduos apresentou renda familiar de 1 até 2 salários mínimos $(82 \% ; n=41)$ e $54 \%(n=27)$ apresentou grau de escolaridade de ensino fundamental incompleto; em relação à caracterização clínica, a presença de comorbidades foi detectada em 94\% (n=47) da amostra, sendo as doenças mais prevalentes HAS $(82 \% ; n=41)$, DM2 (48\%; $n=24)$, doença cardiovascular $(10 \% ; n=5)$ e câncer $(6 \%$; $=3)$, conforme disposto na Tabela 1 .

Tabela 1: Perfil socioeconômico, demográfico e descrição das comorbidades de pacientes renais em tratamento hemodialítico atendidos em um hospital público de referência no Pará, 2019.

\begin{tabular}{|c|c|c|c|}
\hline & & $\mathbf{n}$ & $\%$ \\
\hline \multirow{2}{*}{ Gênero } & Masculino & 25 & 50 \\
\hline & Feminino & 25 & 50 \\
\hline \multirow{3}{*}{$\begin{array}{l}\text { Renda } \\
\text { (salário mínimo) }\end{array}$} & $<1$ & 4 & 8 \\
\hline & 1 a 2 & 41 & 82 \\
\hline & $>2$ até 3 & 5 & 10 \\
\hline \multirow{5}{*}{ Escolaridade } & Analfabeto & 7 & 14 \\
\hline & Ensino Fundamental Incompleto & 27 & 54 \\
\hline & Ensino Fundamental Completo & 5 & 10 \\
\hline & Ensino Médio Incompleto & 3 & 6 \\
\hline & Ensino Médio Completo & 8 & 16 \\
\hline \multirow{4}{*}{ Comorbidades } & Hipertensão arterial sistêmica & 41 & 82 \\
\hline & Diabetes mellitus tipo 2 & 24 & 48 \\
\hline & Doença Cardiovascular & 5 & 10 \\
\hline & Câncer & 3 & 6 \\
\hline
\end{tabular}

Fonte: Autores.

Com relação à análise dos parâmetros bioquímicos, observa-se que as médias dos valores de creatinina $(6,7 \mathrm{mg} / \mathrm{dL})$, hemoglobina $(10,2 \mathrm{~g} / \mathrm{dL})$, cálcio sérico $(1,7 \mathrm{mg} / \mathrm{dL})$ e hematócrito $(37,6 \%)$ encontravam-se abaixo dos valores de referência. Por sua vez, os valores médios de ureia após a hemodiálise $(43,1 \mathrm{mg} / \mathrm{dL})$, potássio sérico $(6,4 \mathrm{mEq} / \mathrm{L})$, ferritina $(451,3 \mathrm{mg} / \mathrm{dL})$ e glicemia $(140,9 \mathrm{mg} / \mathrm{dL})$, apresentavam-se acima dos valores de referência. 
Tabela 2: Caracterização do perfil bioquímico de pacientes renais em tratamento hemodialítico atendidos em um hospital público de referência no Pará, 2019.

\begin{tabular}{|c|c|c|c|c|}
\hline & Indicador Avaliado & Mínimo & Máximo & $\begin{array}{c}\text { Média } \pm \text { Desvio } \\
\text { Padrão }\end{array}$ \\
\hline \multirow{4}{*}{$\begin{array}{l}\text { Exames com valores abaixo do } \\
\text { recomendado }\end{array}$} & Creatinina $(\mathrm{mg} / \mathrm{dL})$ & 2,7 & 13,2 & $6,7 \pm 2,2$ \\
\hline & Hematócrito (\%) & 3,0 & 322,0 & $37,6 \pm 42,1$ \\
\hline & Hemoglobina (g/dL) & 3,0 & 14,5 & $10,2 \pm 2,1$ \\
\hline & Cálcio* (mg/dL) & 1,0 & 19,0 & $1,7 \pm 2,7$ \\
\hline \multirow{4}{*}{$\begin{array}{l}\text { Exames com valores acima do } \\
\text { recomendado }\end{array}$} & Uréia Pós** (mg/dL) & 6,3 & 87,0 & $43,1 \pm 19,5$ \\
\hline & Potássio* (mEq/L) & 3,2 & 51,0 & $6,4 \pm 6,5$ \\
\hline & Ferritina (mg/dL) & 31,3 & 1281,9 & $451,3 \pm 314,5$ \\
\hline & Glicemia (mg/dL) & 49,0 & 398,0 & $140,9 \pm 93,9$ \\
\hline \multirow{5}{*}{ Exames com valores adequados } & Albumina $(\mathrm{g} / \mathrm{dL})$ & 3,0 & 480,0 & $14,7 \pm 67,4$ \\
\hline & Ferro* (mcg/dL) & 27,0 & 154,0 & $66,0 \pm 32,1$ \\
\hline & UréiaPré** (mg/dL) & 81,0 & 220,0 & $150,4 \pm 34,6$ \\
\hline & Fósforo* (mg/dL) & 1,8 & 33,9 & $5,3 \pm 4,5$ \\
\hline & Proteínas Totais (g/dL) & 2,7 & 8,8 & $7,2 \pm 1,2$ \\
\hline
\end{tabular}

*Sérico **Dialise. Fonte: Autores.

Com relação à classificação do IMC, observa-se na Tabela 3, que houve média de 23,9 kg/m² $( \pm 4,7)$. Sendo observada prevalência de eutrofia $(56 \% ; n=28)$, seguida de sobrepeso $28 \%(n=14)$ e apenas $5(10 \%)$ participantes apresentavam algum grau de obesidade. No que diz respeito aos demais parâmetros antropométricos avaliados, observa-se ainda uma média de $27 \mathrm{~cm}( \pm$ $4,1)$ para $\mathrm{CB}$, sendo classificados em baixa musculatura, e média de $2,5 \%( \pm 0,8)$ de GPID.

Tabela 3: Perfil antropométrico de pacientes renais em tratamento hemodialítico atendidos em um hospital público de referência no Pará, 2019.

\begin{tabular}{lcccc}
\hline \multicolumn{1}{c}{ Parâmetro Antropométrico } & n & $\%$ & Média \pm DP & Intervalo \\
\hline Circunferência do braço (cm) & - & - & $27,0 \pm 4,1$ & $20,0--35,0$ \\
\hline Ganho de peso interdialítico (\%) & - & - & $2,5 \pm 0,8$ & $1,0--3,0$ \\
\hline IMC $\left(\mathrm{Kg} / \mathrm{m}^{2}\right)$ & 1 & 2,0 & $16,3--37,2$ \\
Desnutrição moderada & 2 & 4,0 & \\
Desnutrição leve & 28 & 56,0 & \\
Eutrofia & 14 & 28,0 & \\
Sobrepeso & 3 & 6,0 & \\
Obesidade grau I & 2 & 4,0 & \\
Obesidade grau II & & \\
\hline
\end{tabular}

Fonte: Autores.

Quanto a ingestão alimentar, observa-se na tabela 4 que a amostra estudada apresentou ingestão calórica média de 1.806,8 Kcal/dia $( \pm 409,9)$. Em relação à distribuição dos macronutrientes, identificou-se consumo médio de $231,0 \mathrm{~g}( \pm 65,4)$ de carboidratos, 87,7g $( \pm 30,3)$ de proteínas e 64,9g $( \pm 19,5)$ de lipídios do valor energético total (VET). Sendo observado consumo elevado de proteínas por quilo de peso corporal dos pacientes $(1,46 \mathrm{~g} / \mathrm{kg})$ e consumo adequado para carboidratos e lipídios.

Em relação aos micronutrientes, houve um consumo médio diário de 1710,2 mg $( \pm 520,3)$ de potássio e de 578,4mg $( \pm$ 163,9) de cálcio, estando abaixo dos valores recomendados. Observou-se ingestão acima do recomendado, apenas quando 
avaliado a quantidade de ferro 26,9mg $\pm(86,8)$. Quanto aos demais micronutrientes (fósforo e sódio), os valores médios diários estavam em conformidade com as recomendações nutricionais.

Tabela 4: Caracterização do consumo alimentar de pacientes renais em tratamento hemodialítico atendidos em um hospital público de referência no Pará, 2019.

\begin{tabular}{lccc}
\hline & Média \pm DP & Intervalo & Media/kg/dia e \% VET \\
\hline Energia (kcal) & $1806,8 \pm 409,9$ & 603,0 --- 2458,3 & $30,11 \mathrm{kcal}$ \\
Carboidratos (g) & $231,0 \pm 65,4$ & 80,7 --- 404,7 & $51,14 \%$ do VET \\
Proteínas (g) & $87,7 \pm 30,3$ & 20,0 --- 147,1 & $1,46 \mathrm{~g}$ \\
Lipídios (g) & $64,9 \pm 19,5$ & 18,0 --- 103,9 & $32,32 \%$ do VET \\
Potássio (mg) & $1710,2 \pm 520,3$ & 699,0 --- 2677,5 & - \\
Fosforo (mg) & $958,8 \pm 347,5$ & $301,1---1588,3$ & - \\
Sódio (mg) & $1998,9 \pm 725,4$ & $687,8---3925,7$ & - \\
Ferro (mg) & $26,9 \pm 86,8$ & 0,0 --- 610,1 & - \\
Cálcio (mg) & $578,4 \pm 163,9$ & $154,8---921,1$ & - \\
\hline
\end{tabular}

Fonte: Autores.

Quando analisado a correlação entre o GPID com níveis séricos de albumina, observou-se correlação negativa estatisticamente significativa $\left(r^{2}=-0,270 ; p=0,029\right)$, ou seja, quanto maior o GPID, menor os níveis de albumina. Por sua vez, os valores de potássio $\left(\mathrm{r}^{2}=0,245 ; \mathrm{p}=0,043\right)$, fósforo $\left(\mathrm{r}^{2}=0,240 ; \mathrm{p}=0,047\right)$ e cálcio $\left(\mathrm{r}^{2}=0,268 ; \mathrm{p}=0,03\right)$ apresentaram correlação positiva estatisticamente significativa com o GPID, portanto, quanto maior o GPID, maiores os teores séricos destes parâmetros (Tabela 5).

Quando avaliado a correlação entre o IMC e os parâmetros bioquímicos, observou-se correlação positiva estatisticamente significativa apenas para os parâmetros de hematócrito $\left(r^{2}=0,320 ; p=0,012\right)$ e glicemia $\left(r^{2}=0,303 ; p=0,016\right)$. Em relação ao GPID e consumo alimentar, observou-se correlação positiva estatisticamente significativa apenas para o potássio $\left(\mathrm{r}^{2}=0,247 ; \mathrm{p}=\right.$ 0,042), o que demonstra uma relação direta entre estes parâmetros, ou seja, a alteração ocorre de modo proporcional (Tabela 5).

Tabela 5: Correlação entre os dados analisados de pacientes renais em tratamento hemodialítico atendidos em um hospital público de referência no Pará, 2019.

\begin{tabular}{llc}
\hline Correlação & $\boldsymbol{\rho}^{\mathbf{2}}$ & p-valor $^{\mathbf{a}^{\mathbf{0}}}$ \\
\hline Ganho de peso interdialítico $(\mathrm{kg})$ & & 0,029 \\
\hline Albumina $(\mathrm{g} / \mathrm{dL})$ & $-0,270$ & 0,043 \\
\hline Potássio sérico $(\mathrm{mEq} / \mathrm{L})$ & 0,245 & 0,047 \\
\hline Fósforo sérico $(\mathrm{mg} / \mathrm{dL})$ & 0,240 & 0,03 \\
\hline Cálcio sérico $(\mathrm{mg} / \mathrm{dL})$ & 0,268 & 0,042 \\
\hline Potássio dietético $(\mathrm{mg})$ & 0,247 & 0,012 \\
\hline Índice de Massa Corporal $\left(\mathrm{kg} / \mathrm{m}^{2}\right)$ & & 0,016 \\
\hline Hematócrito $(\%)$ & 0,320 & 0,303 \\
\hline Glicemia $(\mathrm{mg} / \mathrm{dL})$ & 0 & 0 \\
\hline
\end{tabular}

a Teste de Correlação de Spearman. Fonte: Autores.

\section{Discussão}

O presente estudo analisou o estado nutricional de pacientes renais crônicos em tratamento hemodialítico atendidos em um hospital público de referência no estado do Pará. Por meio desta pesquisa é possível discutir parâmetros antropométricos e 
bioquímicos de baixo custo e usuais na prática clínica que podem auxiliar a traçar o perfil nutricional desses pacientes, contribuindo para uma prescrição dietética melhor sucedida.

Observou-se distribuição semelhante entre os gêneros feminino e masculino, semelhante ao estudo de Coelho et al. (2018), que avaliou 52 pacientes com doença renal crônica. Entretanto, resultado diferente foi observado por Santos et al. (2018), no qual os autores avaliaram mais de 190 pacientes e observaram prevalência do sexo masculino. Tais achados podem ser justificados pela diferença no tamanho amostral.

Com relação à média de idade, os resultados observados corroboram com estudo de Santos et. al (2018), que apresentou maior prevalência de adultos jovens e idosos em programas de diálise. De acordo com o último Inquérito Brasileiro de Diálise Crônica, a média de idade de 50 a 68 anos, está dentro da faixa de maior risco para ocorrência da doença, uma vez que, quanto maior a idade, maior a probabilidade do indivíduo de possuir agregação dos fatores de risco para o desenvolvimento da doença como diabetes, hipertensão, cardiopatia e obesidade (Santos et al., 2018).

A respeito da escolaridade, a maioria dos pacientes apresentou ensino fundamental incompleto. Segundo um estudo realizado no Brasil, esta associação é esperada, uma vez que a baixa escolaridade está associada com menor nível de conhecimento a respeito da gravidade de doenças crônicas, assim, indivíduos com menor escolaridade chegam ao atendimento de saúde em estado debilitado, com menor chance de reversão do quadro de falência renal (Nerbass et al., 2017).

No que diz respeito à renda, a maioria dos pacientes possuía renda de 1 a 2 salários mínimos, semelhante ao observado no estudo de Caproni et al. (2021) no qual se identificou prevalência de indivíduos com renda de até um salário mínimo. A pobreza potencializa o desenvolvimento de outras doenças crônicas associadas à DRC, com piora de prognóstico (Caproni et al., 2021).

Os achados da prevalência de comorbidades estão em conformidade com outros estudos em que se evidencia maior prevalência de HAS e DM2 (Nerbass et al., 2017, Santos et al., 2018). De acordo com a Sociedade Brasileira Nefrologia (2019), estas duas comorbidades são as principais desencadeadoras da DRC.

No que diz respeito aos parâmetros bioquímicos, com relação aos valores de hematócrito, os achados deste estudo corroboram com o estudo de Oliveira (2021), o qual os autores tiveram como objetivo verificar a ocorrência de anemia em pacientes com DRC que realizavam hemodiálise em um hospital da Região Sul do Brasil, e observaram que 87\% da amostra encontravam-se com os valores de hematócrito abaixo dos parâmetros de referência. O valor de hematócrito é uma medida que permite a verificação das proporções de hemácias no sangue, assim, quando os seus níveis estão abaixo dos valores de referência, há uma redução de hemácias e quando esta redução é acompanhada de redução dos valores de hemoglobina, pode ser indicador de anemia. No que se refere à hemoglobina, assim como nos resultados deste estudo, a pesquisa realizada por Oliveira (2021), identificou que 59,61\% da amostra apresentava valores abaixo da normalidade.

Segundo Oliveira (2021) é comum pacientes portadores de DRC apresentarem quadro de anemia, sendo esta atribuída a um déficit de eritropoietina, que é um hormônio necessário à produção de hemoglobina e apresenta sintetização deficiente após a lesão renal. Além disso, pode ainda ter como agravante a deficiência de ferro que, por sua vez, pode ser ocasionado por inúmeros fatores, tais como desnutrição, redução da ingestão alimentar deste mineral ou ainda se supõem que sua redução se deve às perdas gastrointestinais imperceptíveis, além de perdas na diálise. Assim, é imprescindível a realização de um diagnóstico preciso do tipo de anemia, além de sua real existência, seja por fatores dietéticos ou inerentes ao tratamento, de forma que possa ser realizada conduta precoce, visto que esta apresenta efeitos deletérios ao organismo, principalmente sobre os sistemas cardiovascular e nervoso, além de apresentar relação com a progressão da doença renal (Lima et al., 2020).

Em relação aos níveis séricos de ferritina, estes se encontravam elevados, semelhante aos achados do estudo de Lima et al. (2020) em que se verificou que 60\% do total amostral possuíam ferritina acima dos valores de normalidade. Oliveira (2021) sugerem que níveis elevados de ferritina estejam associados à deficiência funcional de ferro, ou seja, apesar do consumo 
adequado de ferro, o organismo não consegue realizar a sua correta absorção. Um estudo evidenciou que níveis séricos de ferritina, acima do recomendado, podem agravar o estresse oxidativo, em pacientes em HD, contribuindo assim para aumento do risco de complicações cardiovasculares nestes pacientes (Nerbass et al., 2017).

Quanto à ureia pós-diálise, observou-se valores elevados semelhantes ao resultado encontrado no estudo de Oliveira (2021) no qual os autores encontraram valores elevados em $87 \%$ da amostra. De acordo com Nerbass et al. (2017), a diálise tem como objetivo realizar a filtração destes solutos, mantendo os mesmo em quantidades adequadas e não tóxicas ao organismo, portanto, os valores elevados de ureia pós-diálise podem estar associados à dialise ineficiente e presença de estresse oxidativo, o que reforça a necessidade de monitorar esse marcador na prática clínica.

Em relação à creatinina, o presente estudo apresentou resultados semelhantes ao observado por Weissheimer et al. (2021) em que 54,76\% da amostra apresentava valores de creatinina abaixo dos valores de normalidade. De acordo com Oliveira (2021), a creatinina sérica constitui-se em um importante marcador nutricional, de forma que níveis abaixo dos valores de normalidade indicam a redução de massa magra, estando desta forma, associada à elevada taxa de mortalidade, principalmente em pacientes hemodialítico.

A respeito da análise dos níveis séricos de cálcio e potássio, foram encontrados valores abaixo da normalidade, resultados divergentes ao estudo de Weissheimer et al. (2021) no qual os autores evidenciaram adequação em $61,54 \%$ de cálcio e $80,77 \%$ de potássio. De acordo com Weissheimer et al. (2021) é imprescindível manter os níveis de cálcio sérico adequado, principalmente em renais crônicos hemodialíticos, uma vez que possibilita a prevenção da deposição de compostos de cálcio em tecidos moles/saudáveis, impedindo a calcificações vasculares, além de evitar o hiperparatiroidismo secundário. Quanto ao potássio, em pacientes em tratamento hemodialítico, é comum observar desequilíbrio homeostático, com valores acima ou abaixo dos parâmetros de normalidade, que pode ocasionar arritmias cárdicas, com consequente morte súbita (Nerbass et al., 2017).

A respeito da glicose, o presente estudo apresentou valores acima da recomendação, semelhante ao observado no estudo de Nerbass et al. (2017), no qual os autores avaliaram o estado nutricional e composição corporal, em relação ao ganho de peso interdialítico, de pacientes em hemodialise. Foi evidenciado média de $102 \pm 37 \mathrm{mg} / \mathrm{dL}$ da amostra possuía glicose igual ou maior que $106,3 \mathrm{mg} / \mathrm{dL}$.

Quando há excesso de glicose sanguínea, mesmo com excreção urinária aumentada, esta é convertida em sorbitol, depositando-se em tecidos, túbulos renais e nos glomérulos, ocasionando lesão tecidual e depletando o meio intracelular de mioinositol, contribuindo, consequentemente, para níveis elevados de diacilglicerol (DAG), que está relacionado a ativação da proteína quinase C, envolvida na patogênese da nefropatia diabética e progressão da doença renal (Weissheimer et al., 2021). Dessa forma, é necessário monitorar este parâmetro para prevenir complicações clínicas, especialmente em pacientes com diabetes mellitus.

No que se refere à avaliação antropométrica, quando avaliado a classificação do IMC, o resultado obtido neste estudo corrobora com os achados de Santos et al. (2021) no qual 50\% dos participantes estavam eutróficos. Além disso, Rosa (2018) também encontrou IMC médio de $23,6 \pm 4,3 \mathrm{~kg} / \mathrm{m}^{2}$, classificando a maioria dos indivíduos avaliados em eutrofia. De acordo com Santos et al. (2021), o IMC acima de $23 \mathrm{~kg} / \mathrm{m}^{2}$ está relacionado com menor risco para morbimortalidade em pacientes que estão em terapia hemodialítica.

O estado nutricional adequado identificado neste estudo pode representar um serviço nutricional satisfatório, com adequada ingestão energética e tratamento clínico bem sucedido. $\mathrm{O} 1^{\circ} \mathrm{Censo}$ do Estado Nutricional de Pacientes em hemodiálise realizado pela Sociedade Brasileira de Nefrologia (2019) demonstrou que 44,6\% dos pacientes avaliados em tratamento hemodialítico, oferecido pelo Sistema Único de Saúde (SUS), apresentavam com estado nutricional eutrófico, quando comparado com os pacientes atendidos pelo convênio. Contudo, é evidente que o IMC isolado não reflete um quadro avaliativo sensível ao perfil nutricional, tais como a depleção proteica, aumento de tecido adiposo e edema, ou seja, é necessário um conjunto de 
parâmetros nutricionais, com acompanhamento e monitoração periódica para prevenção e diagnóstico inicial de desnutrição (Rosa 2018; Santos et al., 2021).

Em relação à CB, observou-se resultado semelhante ao encontrado por Santos et. al (2021), no qual os autores encontraram como média da CB um valor igual a 27,90 $\pm 3,53 \mathrm{~cm}$, sendo classificados com depleção. Tal classificação pode ser decorrente do processo catabólico ocorrido na terapia de hemodiálise, que degrada o estado nutricional, principalmente as reservas proteicas.

Quanto ao percentual relativo ao GPID, observou-se média dentro da recomendação. De acordo com Brandão et al. (2021) este parâmetro é considerado um componente importante para avaliação do paciente hemodialítica pois, a partir deste, é realizada a avaliação da ingestão hídrica e eficiência da filtração hemodialítica. Além disso, está correlacionado também a condição clínica, uma vez que quando se encontra inadequado, pode sugerir risco para HAS, insuficiência cardíaca congestiva, hiponatremia e alterações clinicas relacionadas a alta eficiência de remoção de fluidos na hemodiálise, levando a hipotensão, angina, arritmia e câimbras (Santos et al., 2021).

Segundo Nerbass et al. (2017) há um importante impacto na relação do GPID com o consumo alimentar e hídrico, à medida que a ingestão de determinados nutrientes, como o de sódio e de líquidos, modifica o percentual de GPID podendo deteriorar o estado clinico e nutricional desta população. Entretanto, são necessários mais estudos a respeito do GPID ser um indicador nutricional para pacientes em hemodiálise.

A respeito da ingestão alimentar, o consumo de energia, carboidratos e lipídios estava em conforme com a recomendação para estes pacientes, a adequação desses nutrientes é considerada como um fator fundamental para melhorar a qualidade de vida e potencializar a sobrevida dos pacientes com insuficiência renal, refletindo em um estado nutricional adequado, assim como demonstrado neste estudo (Werneque et al., 2019).

Observou-se que houve inadequação apenas no consumo de proteínas, em relação aos macronutrientes, nos quais foram observados valores elevados em relação ao recomendado. Diferente do observado por Felix, Medeiros e Molina (2018) no qual os autores encontraram resultados adequados para o consumo proteico $(1,4 \pm 0,5 \mathrm{~g} / \mathrm{kg})$. $\mathrm{O}$ alto consumo de proteína acarreta implicações clínicas, devido ao aumento da oxidação dos aminoácidos, propiciando acúmulo de componentes nitrogenados como a ureia, intensificando a degradação funcional dos rins e prejudicando a eficácia das seções de hemodiálises, pois a elevação de ureia provoca sintomas como náuseas, vômitos e falta de apetite, além de causar síndrome urêmica. Entretanto, o consumo alimentar hiperprotéico nestes pacientes é importante devido ao catabolismo proteico provocado pelo tratamento, sendo necessário o equilíbrio da ingestão (Felix et al., 2018). Devido ao contraste entre os valores de CB e o consumo elevado de proteínas, vale ressaltar a importância de estudos com parâmetros antropométricos com finalidade de analisar massa muscular e de gordura.

Quanto ao consumo de micronutrientes, observou-se consumo elevado de ferro, acredita-se que esse dado seja em virtude do elevado consumo de proteínas observado na amostra. Uma pesquisa realizada com pacientes submetidos à hemodiálise regular Werneque et al. (2019), obteve resultados divergentes, evidenciando consumo adequado de ferro, com média de $14 \pm 05 \mathrm{mg}$. $\mathrm{O}$ ferro é um mineral importante para a formação do sangue, presente nas proteínas animais e em alguns vegetais, o alto consumo desses alimentos fontes de ferro são também alimentos fontes de proteínas e outros micronutrientes que precisam de cautela em sua ingestão, sendo necessário consumo equilibrado. Não foi encontrada na literatura atual a implicação da elevação do ferro alimentar nestes pacientes, cabe assim maiores pesquisa a respeito desde assunto.

Com relação aos valores abaixo do recomendado dos micronutrientes, destaca-se o consumo de potássio e cálcio dietético, esses achados corroboram com o estudo de Werneque et al. (2019) que obteve valores de 1332,39 $\pm 764,88 \mathrm{mg}$ de potássio e 388,12 \pm 401,44 mg de cálcio. O estudo de Felix et al., (2018) analisou o consumo médio diário de desses micronutrientes e observaram valores de 1281,03 $\pm 1141,61 \mathrm{mg}$ e 510,77 $\pm 316,11 \mathrm{mg}$, respectivamente, estando inferiores ao da recomendação para esta população. 
Ao que concerne sobre o consumo de potássio, este deve ser controlado em pacientes em hemodiálise, pois a elevação deste micronutriente acarreta em complicações clinicas severas, como arritmia cardíaca e entre outros riscos cardiovasculares, toda via a adequação é importante para o paciente, para o seu funcionamento muscular e para as células nervosas, evitando a fraqueza ou cãibras musculares ou a diminuição dos batimentos cardíacos (Felix et al., 2018).

Quanto ao consumo de cálcio, é notório o baixo consumo deste mineral, devido os alimentos fontes serem os mesmo que contém fósforo em níveis satisfatórios. Este micronutriente é importante, não apenas para a composição óssea, mas para regulação metabólica e contração muscular, aos quais o cálcio faz-se necessário para a sua adequada funcionalidade. Por essa razão não deve haver uma diminuição deste componente na dieta, sendo recomendado realizar suplementação (Felix et al., 2018).

A respeito das correlações encontradas, observou-se correlação negativa quando avaliado o \%GPID em relação a nível sérico de albumina, semelhante ao do estudo de Luz et al. (2017) no qual justificam que a relação inversa entre estes dois parâmetros pode ocorrer em virtude da função da albumina em manter o equilíbrio da pressão osmótica.

Verificou-se relação positiva entre o aumento do \%GPID e concomitante aumento de fósforo, cálcio e potássio sérico, sendo este resultado semelhante o estudo de Nerbass et al. (2017) De acordo com os autores, este achado se justifica em virtude da dificuldade destes pacientes em atender às orientações dos nutricionistas, assim, os pacientes que apresentam dificuldade no controle da ingestão hídrica tendem a apresentar descontrole no consumo de alimentos com elevado conteúdo de fósforo, cálcio e potássio.

Quando avaliado o IMC em relação aos parâmetros bioquímicos (hematócrito e glicemia), encontrou-se correlação positiva. No estudo de Soares et al. (2017) a relação entre o IMC e o hematócrito não foi significativa. Entretanto, com relação à glicemia, no estudo de Nerbass et al. (2017) observou-se que o aumento do corporal é proporcional ao aumento da glicemia, por serem derivados do consumo inadequado de energia e carboidratos, que elevam tanto o IMC quanto a glicemia, predispondo à resistência insulínica e aparecimento ou piora do quadro de DM2, assim como a diminuição de 5 a $10 \%$ do peso corporal diminui proporcionalmente os níveis de glicemia e melhora da resistência insulínica (Nerbass et al., 2017).

Quando testada a associação entre o \%GPID e o potássio dietético, verificou-se relação positiva significativa. No estudo de Nerbass et al. (2017) os autores retratam que em 30\% dos pacientes possuem prevalência de alterações proporcionais entre esses dois componentes, pois as dificuldades na adesão às restrições dietéticas provocam acúmulo de produtos metabólicos e excesso de fluídos no sistema circulatório, ocasionando risco elevado de morbimortalidade destes pacientes, ou seja, a inadequação da restrição dietética do potássio provoca aumento do peso interdialítico devido à alteração dos fluidos corporais.

A inclusão unicamente de indivíduos de uma mesma clínica constitui-se na principal limitação desde estudo, assim os dados dessas pesquisas não podem ser realizados para representar toda a região Norte do País, visto que existem outras clinicas de referência nessa região.

\section{Considerações Finais}

Desde modo, o perfil clínico dos pacientes renais crônicos encontra-se alterados com relação aos dados bioquímicos, enquanto ao estado nutricional mostrou-se adequado pela análise de IMC, contudo quando avaliado os valores de CB verificou depleção proteica advindo dos processos metabólicas do tratamento hemodialítico, sobre a avaliação dietética evidenciou-se elevado consumo proteico e ferro, e baixo consumo de cálcio e potássio.

Este estudo reforça as evidências de que não há um único parâmetro que forneça resultados completos a respeito do estado nutricional, assim faz-se necessário a associação entre diversos indicadores tais como antropométricos, bioquímicos, dietéticos e clínicos para que se verifiquem os riscos ou anormalidades nutricionais já instalados ou de possível instalação, possibilitando assim a correta intervenção nutricional. 
É importante ressaltar que foram incluídos somente indivíduos de uma mesma clínica, consequentemente não é possível estender os resultados encontrados às demais clínicas, portanto, sugerem-se a necessidade de novos estudos a fim de se verificar se estes achados são um padrão. Além disto, sugere-se que sejam realizados estudos longitudinais para que seja verificada a adesão ao tratamento nutricional por estes pacientes.

\section{Referências}

Alvarenga, L. D. A., Andrade, B. D., Moreira, M. A., Nascimento, R. D. P., Macedo, I. D., \& Aguiar, A. S. D. (2017). Análise do perfil de pacientes renais sensíveis ao tratamento nutricional em relação ao tempo de tratamento. Revista Brasileira de Nefrologia, 39, $283-286$.

Brandão, H. F. C., Saraiva, M. B. M., de Sousa, B. S., de Almeida, S. S., Souza, E. D. S., Melo, H. C. M., \& Tomyia, M. T. O. (2021). Estado nutricional e sua associação com risco cardiovascular no paciente em tratamento hemodialítico. Brazilian Journal of Development, 7(2), 11712-11728.

Butyn, G., de Carvalho, G. M., de Castro, C. J. S., da Silva, G. R., Arcaro, G., Martins, C. M., \& Mikowski, J. R. D. (2021). Avaliação da qualidade de vida do paciente com doença renal crônica em terapia renal substitutiva. Brazilian Journal of Health Review, 4(1), $2785-2798$.

Caproni, L. M. E., Madeira, L. A., \& Santos, G. B. (2021). Impacto da insuficiência renal em regiões de baixa renda. RECIMA21-Revista Científica Multidisciplinar-ISSN 2675-6218, 2(7), e27527-e27527.

Coelho, P. E. F. S., Gomes, F. A. R., Neves, C. V. B., \& Alves, N. E. G. (2018). Perfil dos parâmetros bioquímicos em pacientes com doença renal crônica submetidos à hemodiálise. Ágora, 1, 62-74.

Felix, L. C. M., Medeiros, V. C. M. D., \& Molina, V. B. C. (2018). Análise do conhecimento e consumo de alimentos fontes de fósforo por pacientes portadores de insuficiência renal crônica em tratamento dialítico. Braspen J, 33(1), 15-20.

Lima, R. S. G., de Souza Lima, M., de Lima, L. S. G., Soares, M. D. S. C., Vasconcelos, A. C. M., Nasserela, J. C. L., \& da Silva, L. R. F. (2020). Perfil hematológico e bioquímico de pacientes renais crônicos em tratamento hemodialítico. DêCiência em Foco, 4(1), 132-139.

Luz, C. A., Cortes, M. L., Souza, J. S., Gomes, L. F. O., Alves, A. B., \& Cairo, I. G. (2017). Avaliação da ingestão nutricional de pacientes com doença renal crônica em tratamento hemodialítico. Braspen J, 241-245.

Nerbass, F. B., Correa, D., Santos, R. G. D., Kruger, T. S., Sczip, A. C., Vieira, M. A., \& Morais, J. G. (2017). Percepções de pacientes hemodiálise sobre as acolhidas em alimentos. Revista Brasileira de Nefrologia, 39, 154-161.

Oliveira, B. R. D. O., Coutinho, A. L. N., Lopes, J. M., Tenório, A. P. D. O., \& Tenório, P. P. (2021). Adesão de pacientes com doença renal crônica à hemodiálise. Rev. Enferm. UFPE on line, 1-16.

Oliveira J. J. S. D. (2021). Perfil clínico-epidemiológico e parâmetros bioquímicos em pacientes com doença renal crônica submetidos a tratamento hemodialítico na região do Médio Araguaia-MT.

Rosa, LND (2018). Estado nutricional de pacientes com doença renal crônica em hemodiálise atendidos em uma clínica renal de Cachoeira do Sul-RS.

Santos, K. K. D., Lucas, T. C., Glória, J. C. R., Pereira Júnior, A. D. C., Ribeiro, G. D. C., \& Lara, M. O. (2018). Perfil epidemiológico de pacientes renais crônicos em tratamento. Rev. enferm. UFPE on line, 2293-2300.

Santos, M. V. R., Figueiredo, R. R. B., de Alcântara, R. V. P., de Almeida, S. S., de Morais, C. N., \& de Melo, M. C. A. L. (2021). Estado Nutricional e Qualidade de Vida de Pacientes com Doença Renal Crônica Submetidos à Hemodiálise: Estado Nutricional e Qualidade de Vida. Nutrición Clínica y Dietética Hospitalaria, 41(4).

Sesso, R. C., Lopes, A. A., Thomé, F. S., Lugon, J. R., \& Martins, C. T. (2016). Inquérito brasileiro de diálise crônica 2014. Brazilian Journal of Nephrology, 38, 54-61.

Soares, S. S., da Silva, C. F., Kern, D. G., Sehn, A. P., Welser, L., Pohl, H. H., \& Burgos, M. S. (2017). Relação entre indicadores antropométricos e hematológicos de pais e seus filhos. Saúde, 43(2), 109-115.

Sociedade Brasileira de Nefrologia (SBN). Censo de diálise 2011. 〈http://www.sbn.org.br/pdf/censo_2011_publico.pdf>. Acesso em: 15 nov. 2019.

Weissheimer, R., Bucharles, S. G. E., Truyts, C. A. M., Jorgetti, V., Figueiredo, A. E., Barrett, P., \& Moraes, T. P. D. (2021). Alta Prevalência de Distúrbios Bioquímicos Minerais e Ósseos na Doença Renal Crônica em uma coorte de diálise peritoneal nacional: as metas das diretrizes são muito difíceis de alcançar? Brazilian Journal of Nephrology.

Werneque, I. C., Neto, W., Perusso, F., Farias, L., Junior, W., \& Silva, F. (2019). Alimentação e hábito de vida na doença renal crônica. Cadernos da MedicinaUNIFESO, 2(2). 